\title{
Figuration graphique et appropriation d'un objet technique
}

\section{Erick Cazalet}

\section{Q OpenEdition \\ 12 Journals}

\section{Édition électronique}

URL : http://journals.openedition.org/trema/2248

DOI : 10.4000/trema.2248

ISSN : 2107-0997

\section{Éditeur}

Faculté d'Éducation de l'université de Montpellier

\section{Édition imprimée}

Date de publication : 1 décembre 1994

Pagination : 53-67

ISSN : 1167-315X

\section{Référence électronique}

Erick Cazalet, «Figuration graphique et appropriation d'un objet technique », Tréma [En ligne], 6| 1994, mis en ligne le 01 décembre 1994, consulté le 03 mai 2019. URL : http://journals.openedition.org/ trema/2248 ; DOI : 10.4000/trema.2248

Ce document a été généré automatiquement le 3 mai 2019.

Trema 


\title{
Figuration graphique et appropriation d'un objet technique
}

\author{
Erick Cazalet
}

1 Dans la pratique quotidienne de la classe, qu'appelle-t-on un « enseignement réussi »?

2 Habituellement, nous disons cela à propos d'une séance où :

1. le déroulement du protocole technique ou expérimental a été entièrement suivi et les manipulations sont toutes accomplies ;

2. l'objet étudié a été suffisamment dépouillé, les lois sous-jacentes en sont extraites, une synthèse interdisciplinaire a été menée ...

3 Si tout a "réussi », cela veut-il dire aussi que les élèves ont intégré tout ce que les objectifs initiaux visaient?

4 Quels sont les effets réels produits sur le plan du fonctionnement cognitif de nos élèves (celui des constructions mentales relatives au domaine des connaissances considérées et celui de la mise en oeuvre des acquisitions cognitives en différentes situations)?

\section{Problématique Présentation de la tâche, population}

5 1.1. La question posée est celle de l'appropriation de l'objet technique chez l'enfant. Par rapport à cette question, la problématique la plus féconde parait correspondre à l'étude de la construction effective de cet objet.

6 A la formation des opérations motrices (actions nécessaires à la construction de l'objet matériel) doivent s'articuler les opérations mentales nécessaires à la compréhension et à la représentation de l'objet. Les connaissances relatives au savoir-faire pratique et au savoir théorique ne s'opposent pas chez l'enfant (Vergnaud, 1977; Bresson, 1987). L'intelligence pratique et l'intelligence rationnelle se soutiennent mutuellement à travers divers processus généraux : adaptation, appropriation, prise de conscience, abstraction (Rey, 1935 ; Piaget, 1974 a et b ; Halbwachs, 1975 ; Inhelder et Cellérier, 1992). Dans la mise en œuvre de ces processus, les aspects figuratifs et opératifs des fonctions cognitives revêtent une importance capitale, par des liens étroitement entretenus avec les 
représentations de type sémiotique (Vergnaud, 1974; Pinol-Douriez, 1975; Inhelder et Cellérier, 1992).

7 Si l'importance et l'utilité des représentations figuratives ne sont plus à démontrer (Weill-Fassina, 1973; Rabardel et Weill-Fassina, 1987) dans les domaines techniques et scientifiques, il reste toutefois à savoir quel est le moment le plus opportun pour que leur introduction apporte une aide maximale au cours de l'apprentissage. C'est pourquoi a été exploré en priorité et de manière plus systématique le rôle de la figuration graphique. Par hypothèse, j'ai rattaché cette influence à un mode particulier de figuration graphique : celle produite par le sujet lui-même, à sa convenance.

$8 \quad 1.2$.Dans le cadre des tâches de résolution de problème, une tâche à caractère technique, susceptible d'entrer dans les programmes d'enseignement du premier cycle $\mathrm{du}$ secondaire a été recherchée.

Mon choix s'est porté sur le va-et-vient électrique. C'est un objet technique complexe et son utilisation facile était connue par tous les élèves qui constituent les populations examinées. Une fonction globale finalise, sans équivoque, ce qui est à faire. Cette fonction sous-tend des fonctions principales simples et limitées en nombre. Chacune correspond à un élément de matériel qui entre en jeu dans la construction. Le contrôle par la mise sous tension, met en évidence la réussite ou l'échec. En outre la figuration graphique de cet objet ne présente pas de difficultés particulières.

Figure 1 : Schéma de principe du va-et-vient électrique.

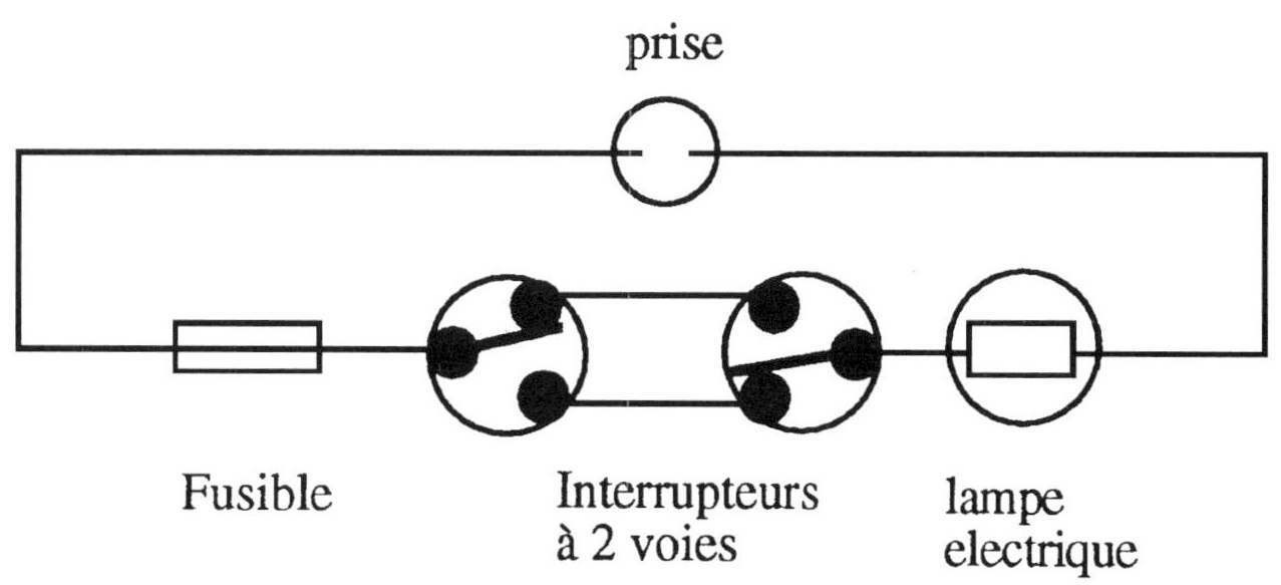

10 Avec le matériel utilisé (Cazalet, 1984 ; Cazalet et Dusseau, 1991) les constructions, les assemblages possibles peuvent engendrer une multitude de cas de figures. Cependant un seul de ces cas possibles et équiprobables répond à la fonction technique globale du va-etvient: "allumer ou éteindre l'ampoule de deux endroits, de deux interrupteurs ». Les autres cas de figures possibles répondent à d'autres fonctions. En outre, il est possible d'interpréter chacun de ces cas possibles dans un cadre plus abstrait : celui d'une table de vérité.

11 Après une présentation sous forme de dialogue de la fonction globale du va-et-vient électrique, du matériel utilisé et de ses fonctions spécifiques ; la tâche demandée au sujet est d'essayer, avec ce matériel, de réaliser le montage qui, selon lui, correspond à la fonction globale du va-et-vient électrique - but de la tâche prescrite (Leplat et Hoc, 1983).

12 En émettant l'hypothèse de l'importance de l'activité de figuration graphique dans la résolution de problèmes techniques, deux types de situations ont été examinés. Dans la 
situation expérimentale, le sujet raisonne à chaque essai sur l'objet qu'il a confectionné en prenant appui sur la figuration graphique qu'il a tracée. Dans la situation contrôle, le sujet ne raisonne que sur l'objet réalisé. En durée les deux situations ne diffèrent pas. Chacune comporte un nombre identique d'essais. A l'intérieur de chaque essai, nous nous sommes efforcé d'analyser les comportements recueillis dans différents items ordonnés comme suit :

1.2.1. à l'item réalisation, le sujet construit l'objet qui lui paraît le mieux correspondre à la solution du problème ;

1.2.2. à l'item explication de la réalisation, le sujet tente de donner les raisons qui l'ont amené à produire tel ou tel assemblage.

Ensuite le contrôle de la fonction globale demandée s'effectue grâce à la mise sous tension. L'essai se poursuit par les items suivants :

16 1.2.3. à l'item explication du contrôle, le sujet tente d'expliquer les effets observés ;

1. dans la situation expérimentale, et seulement dans ce cas, le sujet produit à sa convenance une figuration graphique (item correspondant) de l'objet et tente de raisonner en s'appuyant dessus : c'est l'item explication sur la figuration graphique,

2. dans la situation contrôle, on insiste davantage sur les commentaires seulement verbaux des sujets, c'est-à-dire sans l'appui de la figuration graphique, en vue d'équilibrer en durée les deux situations.

17 Le premier essai terminé, l'expérimentateur invite le sujet à rectifier, à modifier ou à produire une autre réalisation... et le deuxième essai est commencé. L'épreuve s'arrête d'elle-même à la fin de l'essai (item 3) dès que le sujet a trouvé la bonne solution. S'il n'arrive pas à construire, refuse de continuer, l'interruption est immédiate. Ce comportement s'observe en particulier au moment de la réalisation (item 1) et ce quel que soit l'essai.

18 1.3. Trois expérimentations sont réalisées en collège avec des élèves des classes de sixième et de quatrième :

1. quatre-vingts élèves de sixième, âgés de 12 ans \pm 6 mois pour la première expérience ont subi une seule passation contenant trois essais;

2. quarante élèves de quatrième, âgés de 14 ans \pm 6 mois pour la deuxième expérience ont subi deux passations, à deux semaines d'intervalle ; chaque passation contenait deux essais ;

3. vingt élèves de sixième, âgés de 11 ans 10 mois \pm 10 mois ont subi, pour la troisième expérience, un entraînement étalé sur cinq séances contenant deux essais chacune ; chaque séance était séparée de la suivante par un intervalle de deux semaines.

Chaque élève est examiné individuellement, soit environ 260 entretiens. Quelques rares sujets «perdus en cours de route» resteront «inutilisables» pour une analyse approfondie de leurs comportements.

\section{Cotation des résultats}

Elle porte sur les items suivants :

- réalisation - explication - figuration graphique.

\subsection{Item réalisation :}



nombreux montages. Toutefois le nombre de productions des sujets reste réduit. Quatre types de régularités sont observées : le type R0, le type I, le type II, le type III.

2.1.1. Le Type Ro correspond au refus de la part du sujet. Ce refus est motivé :

1. soit par l'impossibilité de réaliser un montage quelconque,

2. soit par un arrêt après une recherche infructueuse menée par tâtonnements.

2.1.2. Le Type I où les constructions réalisées sont sous-tendues par le modèle unipolaire. Il n'y a jamais de circuit fermé et donc l'ampoule n'est jamais éclairée. Il s'agit d'un assemblage sur la base de relations locales où des symétries peuvent apparaître. Le montage le plus fréquemment rencontré est présenté sur la figure 2.

Figure 2 : Modèle unipolaire, base des montages de type I.

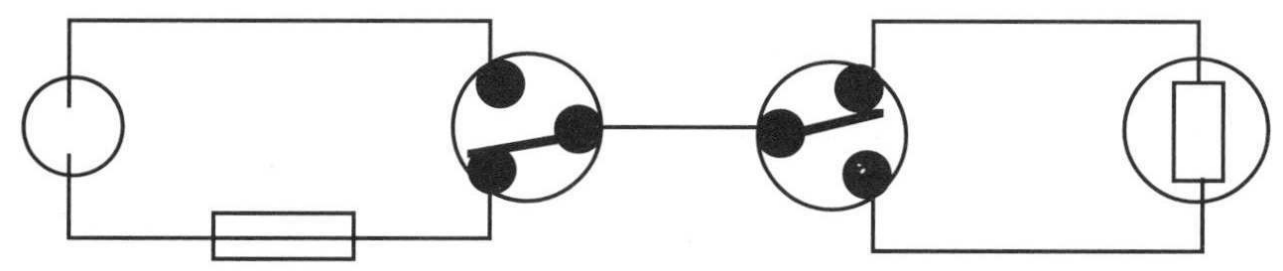

2.1.3. Le Type II où l'ampoule est située entre les deux interrupteurs. Il peut y avoir des symétries bilatérales ou relativement à l'ampoule. La figure 3 présente le montage le plus souvent observé.

Figure 3 : Montage de type II le plus fréquent.

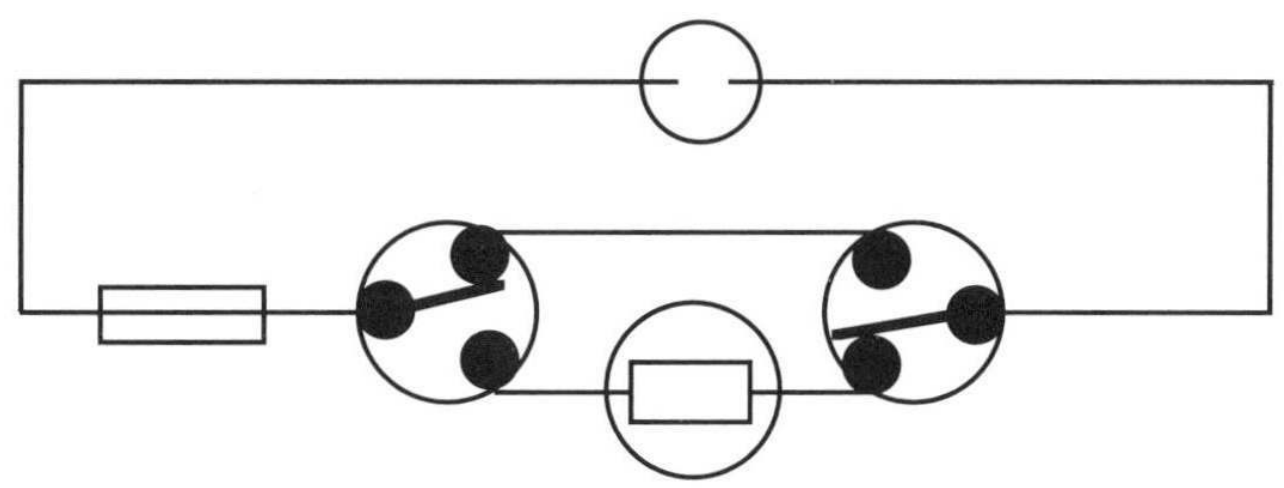

2.1.4. Le Type III oùl'ampoule est située hors des liaisons entre les interrupteurs. Il ne peut y avoir les symétries précédentes. Ces montages sont les résultats de permutations des fils sur une suite de positionnements corrects de l'appareillage. Ce type contient donc le montage exact (Figure 1).

\subsection{Item explication verbale}

Les réponses recueillies sont nombreuses et très diversifiées dans leurs formes. Cependant les contenus peuvent se regrouper en un nombre restreint de catégories. Ces catégories correspondent à différents registres explicatifs hiérarchisés : - anecdotique - descriptif - constat - préformalisé - formalisé -.

31 2.2.1. Le registre anecdotique constitue le niveau le plus bas. Aucune compréhension n'apparaît. 
2.2.2. Le registre descriptif est une présentation des réalisations qui concerne des états présents ou passés du matériel - et non des relations entre ces états. Ces derniers sont jugés indépendants les uns des autres par les sujets.

2.2.3. Le registre de constat est une description qui repose sur des actions effectuées dans une relation strictement locale. Un ou plusieurs faits sont bien localisés, bien décrits ; mais rien n'est relié dans un système d'ensemble. Il s'agit d'un mode de raisonnement intuitif.

registre préformalisé, du moins est-ce une hypothèse que l'on peut émettre..., est l'exposé d'un cas précis avec des mises en relations, précises et exactes, entre un état de l'ampoule et un état des interrupteurs. Mais le sujet ne peut transférer ou généraliser ses explications à tous les états des interrupteurs et de l'ampoule.

2.2.5. Le registre formalisé constitue une verbalisation de la table de vérité du montage confectionné, avec toutes les relations explicitées entre tous les états des interrupteurs et tous les états de l'ampoule.

\subsection{Item figuration graphique}

Évaluer des figurations graphiques est une chose délicate. Une manière efficace et simple de l'aborder consiste à parler en terme de lisibilité ou de clarté de la figuration. Selon Weill-Fassina (1973) « un schéma clair » est un schéma "disposé de telle manière que, même en procédant de proche en proche, les sujets puissent retrouver la structure fonctionnelle ». Cet auteur décrit sept critères qui contribuent à une bonne lisibilité.

Avec les productions graphiques recueillies, j'ai pu élaborer un système de cotation fondé sur les sept critères retenus par l'auteur cité et ce système concerne deux ordres :

1. celui relatif à l'espace : -point de vue -distances et directions relatives.

2. celui relatif au degré de dépouillement de la figuration des fonctions techniques transmissions électriques -reconstructions figuratives de la chaîne électrique.

Relativement aux critères concernant ces deux ordres, une échelle hiérarchique, un scalogramme, a pu être établi. Le coefficient de reproductibilité de Guttman est .925 (Cazalet, 1984).

\section{Résultats quantitatifs, bilan des deux premières expériences}

Il s'agit du premier groupe d'élèves de sixième (80 sujets, 12 ans \pm 6 mois) et du groupe d'élèves de la classe de quatrième ( 40 sujets, 14 ans \pm 6 mois).

Nous analyserons principalement, dans la suite de cet article, les résultats à l'item réalisation.

3.1. L'utilisation des modèles électriques unipolaires (type I) est source d'incompréhensions et d'erreurs. L'utilisation des modèles électriques bipolaires marque un progrès (type II et type III).

3.2. La reconnaissance des montages équivalents -mais perceptiblement différentspose de sérieux problèmes.

$\mathrm{Au}$ cours des essais successifs, certains sujets croient modifier leur réalisation par la simple permutation de deux fils. Seul, l'aspect figuratif varie et il y a conservation (non perçue comme telle) des aspects fonctionnels. Dans le cas de la figure 4 ci-après, les élèves 
ne prennent en compte qu'une partie de la consigne : « allumer ou éteindre l'ampoule... » Ils ne peuvent coordonner les liens entre les deux interrupteurs et ne raisonnent que sur l'un d'eux.

Figure 4 : Modification d'un montage par inversion de deux fils.

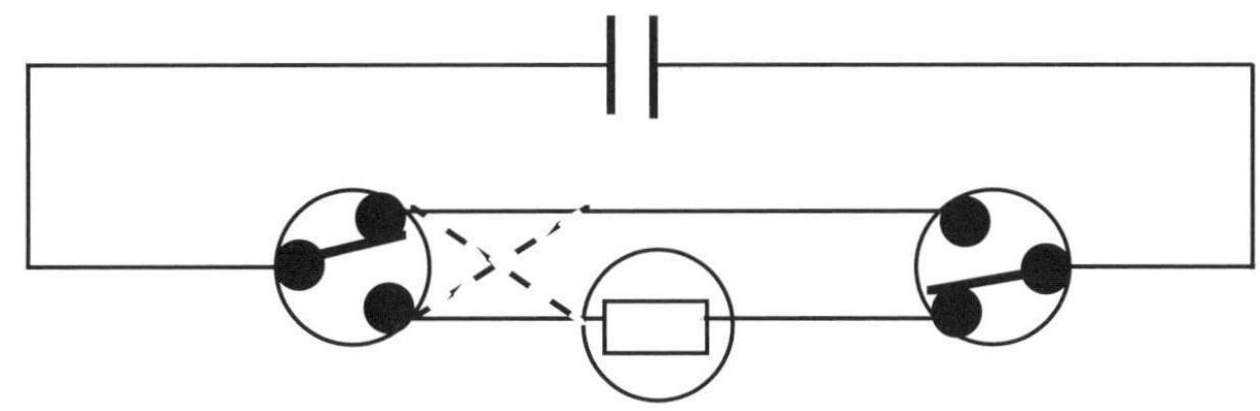

Ce mode de modification des montages par la simple permutation de deux fils est désigné par : «mode répétition ».

Le mode variation interviendra lorsqu'au cours des essais successifs, il y a changement de circuit électrique à l'intérieur du même type ou changement de type. Cela n'est pas obligatoirement un progrès (passage du type II au type I par exemple).

3.3. Le tâtonnement a lieu au cours d'un même essai. C'est l'annonce de processus de recherche avec des tentatives plus ou moins nombreuses de résolution du problème. Dans l'item réalisation, le sujet positionne les éléments de matériels sans les fixer. Il procède ensuite à un contrôle, à un «calcul mental» intériorisé (ou quelquefois évoqué). S'il aperçoit une erreur selon lui, il corrige ou recherche autre chose. Cette tendance au tâtonnement induit fortement la réussite à l'épreuve $(\mathrm{X} 2=6,3$ et le seuil de signification est proche de .01).

\subsection{La production et l'utilisation de la figuration graphique amènent des effets} divers selon la population (toujours analysés à propos de l'item réalisation).

49 A 12 ans ( \pm 6 mois), les groupes qui bénéficient de l'intermédiaire graphique ont tendance à continuer les essais pratiques et les tâtonnements $(\mathrm{X} 2=4,26$, le seuil de probabilité est .05). Les réalisations effectuées s'orientent vers des progrès bien que les réussites ne soient pas massives. Dans le groupe témoin, les sujets abandonnent tôt et ceux qui poursuivent ont tendance à répéter les mêmes montages (mode répétition, $\mathrm{X} 2=3,96$, le seuil de probabilité est .05).

A 14 ans ( \pm 6 mois), un apprentissage scolaire antérieur de la schématisation électrique semble perturber certains sujets. L'utilisation non maitrisée d'éléments d'une schématisation électrique normalisée - ou seulement l'intention de les utiliser - gênent de nombreux sujets pour se situer par rapport à l'objet confectionné. Dans les cas extrêmes, l'objet dessiné est étranger à l'objet réalisé. Ces phénomènes font que les sujets de la situation contrôle (sans intermédiaire graphique) obtiennent de meilleurs résultats que les sujets des groupes expérimentaux (qui produisent et utilisent l'intermédiaire graphique). Le calcul du X2 $(=4,08)$ donne un seuil de probabilité à .05. Dans la situation contrôle, tâtonnements et réussites quoique tardifs dans le cours des essais, sont bien plus fréquents. 

graphique.

\subsection{Objectif}

Cependant, chez les sujets, peu nombreux, qui maîtrisent le langage figural normalisé, tâtonnements et réussites se manifestent au cours des essais plus précocement que chez tous les autres sujets qui réussissent.

3.5. Ces deux expérimentations apportent, sur le rôle des variables secondaires - niveau scolaire - niveau opératoire - sexe - âge - des informations relativement attendues et ce, quel que soit l'item considéré, réalisation ou explications verbales.

Les sujets plus âgés réussissent mieux l'épreuve : les élèves des classes de quatrième ont de bien meilleurs résultats.

Les élèves à bon niveau scolaire ${ }^{1}$ ou à niveau opératoire formel ${ }^{2}$ ont des résultats bien supérieurs aux autres élèves.

Les garçons ont tendance à obtenir des performances meilleures que celles des filles. Cette tendance - non significative au X2 - pour les élèves des classes de sixième, s'amplifie chez les élèves des classes de quatrième .

3.6. Relations entre les items réalisation, explication verbale et figuration

Aucun lien direct n'a pu être observé entre les types de réalisation (type Ro, I, II ou III) et les registres explicatifs (anecdotique, descriptif, constat... ).

Le travail de reconstruction graphique de la réalité perçue, mené par les sujets dans la situation expérimentale conduit aux observations suivantes.

9 Plus la figuration graphique est «claire », plus l'explication donnée tend vers le niveau formalisé. Dans la situation expérimentale, réussir à clarifier la figuration graphique implique au moins une réponse de type préformalisée ou formalisée (X2 = 6,75 le seuil de probabilité est à .01).

3.7. Les indications données par ces deux expériences m'ont incité à dépasser le cadre des activités strictement liées à la seule construction du va-et-vient électrique.

\section{Mise en place de l'expérience d'apprentissage}

Pour mieux saisir la dynamique d'évolution des sujets dans la construction effective des objets et dans les connaissances élaborées, il convient d'analyser le changement et les conditions de changement in vivo (Orsini-Bouichou, Hurtig, Paour, Planche, 1990, p. 223-224). L'introduction d'une procédure d'apprentissage répond à cet objectif. Nous espérons ainsi mieux cerner ce qui se joue à travers la variable figuration graphique.

\subsection{Population}

Pour éviter les difficultés d'interprétation suscitées par l'hétérogénéité des élèves d'une classe scolaire, nous avons recruté une population de bons élèves de la classe de sixième (2) et (3)). C'est au début de l'année scolaire, avant tout enseignement portant sur les montages électriques simples et leurs schématisations, que l'expérience est menée. Ces sujets, au nombre de 20 , sont âgés de 11 ans 10 mois \pm 10 mois.

65 Le problème du va-et-vient électrique est pour ces enfants très difficile s'il est posé sans préalable ni entraînement (Joshua, 1984). Il devient beaucoup plus abordable s'il intervient au terme d'une série de séances d'entraînement. 

expériences, il s'agit maintenant de proposer aux sujets des entraînements progressifs qui leur permettront de résoudre les problèmes posés et d'aboutir à une meilleure réussite.

Ces situations d'entraînement prennent en compte :

1. la définition d'objectifs par rapport au champ conceptuel concerné : celui des circuits électriques élémentaires ;

2. les représentations et les connaissances préexistantes chez les élèves (cf première et deuxième expérience qui apportent ces informations);

3. les conditions de réalisation (rôle de la figuration graphique, mode de travail du sujet) ;

4. la mise en œuvre de planification de l'action (anticiper une série d'actions, évoquer des projets de constructions...).

Il s'agit d'optimiser les savoir et savoir-faire initiaux et de permettre au sujet l'autocontrôle (anticipateurs, rétroactifs) des actions et du choix des opérations à réaliser.

Pour mieux comprendre et comparer les processus d'apprentissage inférés, nous avons conservé les deux modalités conformément à l'hypothèse initiale. Avec un groupe d'enfants, on a recours à la production et à l'utilisation de figurations graphiques en présence de l'objet, sur la demande de l'expérimentateur, mais à la convenance du sujet quant au code graphique. L'autre groupe bénéficie de la même progression mais les questions de l'expérimentateur incitent à raisonner seulement verbalement en présence de l'objet fabriqué.

\section{Déroulement des apprentissages, résultats}

\subsection{L'apprentissage proprement dit est constitué de trois étapes.}

Chaque étape comprend une séance de deux essais maximum. Seule la quatrième étape constituée de deux séances de deux essais est consacrée au va-et-vient électrique. Le matériel est introduit progressivement au cours de l'entraînement en vue d'une meilleure familiarisation. Les consignes sont, elles aussi, graduées en difficultés. Un intervalle de deux semaines sépare deux séances consécutives.

\subsection{1. Étape 1.}

Un seul essai, le simple allumage. Dans cet essai, l'objectif à atteindre est la construction d'un circuit électrique (ou chaîne électrique) avec élimination des montages de type I basés sur l'unipolarité. La consigne est celle du simple allumage : «allumer ou éteindre une ampoule avec un seul interrupteur ». Compte tenu du matériel disponible et de sa présentation, c'est une situation de réussite pratique forcée.

74 5.1.2. Étape 2.

L'objectif de cette étape (deux essais) est la construction de montages équivalents afin de réduire les aspects répétitifs : éviter la rigidité de comportement qui consiste à inverser deux fils pour corriger un montage. La consigne est «éteindre l'ampoule d'un seul des deux interrupteurs, ainsi lorsque l'ampoule est allumée, la manœuvre d'un seul interrupteur suffit à l'éteindre » (les deux interrupteurs doivent être montés en série). Le matériel utilisé est celui de l'étape I, plus un fil et un autre interrupteur. Comme précédemment, c'est une situation de réussite forcée. 


\section{électrique. Cette étape comporte deux séances de deux essais, séparées par deux} semaines d'intervalle.

Avec ce matériel, il y a plusieurs montages correspondant à la consigne. Après le premier essai, il est demandé au sujet de trouver autre chose d'équivalent.

\subsection{3. Étape 3 .}

L'objectif de cette étape (deux essais) est d'amener les sujets à changer de type de montage tout en ayant des montages fonctionnellement équivalents, ce qui conduit à procéder à un inventaire anticipateur des montages à réaliser. Les consignes et le matériel sont les mêmes qu'à l'étape 2 . Il s'agit de positionner l'ampoule en différents endroits du circuit. Les élèves manipulent ainsi la combinatoire du matériel sans perdre de vue les équivalences de montages. A cette étape, nous avons invité les sujets à clarifier leurs expressions par l'introduction d'un aspect " communication à autrui ", au moment de la figuration graphique, propre à la situation expérimentale comme au moment des explications verbales dans la situation contrôle.

Les séances d'entraînement s'arrêtent là.

5.1.4. L'étape 4 qui suit est celle de l'épreuve terminale consacrée au va-et-vient

\subsection{Bilan des résultats.}

Tout au long de l'entraînement, les deux situations (avec ou sans figuration graphique) présentent des résultats parfaitement analogues (item réalisation et item explication).

Mais l'apprentissage médiatisé par le graphisme permet de transférer au problème final les connaissances acquises pendant l'apprentissage précédent.

\subsubsection{Item réalisation :}

1. le modèle unipolaire n'apparaît plus dans la situation expérimentale (plus de montage de type I) ; ce modèle persiste dans la situation contrôle ;

2. un phénomène analogue concerne aussi l'aspect répétition (reconnaissance de montages équivalents) ;

3. la tendance au tâtonnement est plus marquée dans la situation expérimentale (le calcul du T.P.E.F. donne un seuil de probabilité à .05) ;

4. une meilleure approche de la solution au problème du va-et-vient apparaît dans la situation expérimentale: la proportion de montage de type IIIest plus élevée dans la situation expérimentale (le calcul du T.P.E.F. donne un seuil de probabilité à .08), lors du deuxième essai de la première séance. Mais par la suite, l'activité des sujets homogénéise les groupes, particulièrement à la fin de la deuxième séance.

\subsubsection{Item explication.}

Dès le premier essai, dans la situation expérimentale, le niveau des explications reste ce qu'il était pendant les séances d'entraînement - environ 60 à $70 \%$ de réponses formalisées. Il n'en va pas de même dans la situation contrôle où on assiste à une chute du niveau d'explication.

Remarquons que c'est à la suite du premier essai que les tâtonnements apparaissent dans la situation expérimentale (donc après un bon niveau d'explication attestant d'une compréhension plus élaborée du problème).

Remarquons que c'est au même moment qu'apparaît une meilleure approche de la solution au problème dans la situation expérimentale. 
89 avons observé la chose suivante :

1. l'évolution de la figuration graphique vers des formes plus claires au cours de l'apprentissage s'accompagne toujours de la réussite à l'épreuve du va-et-vient ;

2. s'il n'y a pas d'évolution de la figuration graphique vers des formes plus claires (entre l'étape 1 et l'étape 4), il n'y pas réussite à l'épreuve du va-et-vient.

\section{Interprétation des résultats des trois expérimentations}

Les résultats obtenus montrent l'existence de liens étroits entre actions, représentations, figurations graphiques, explications verbales. Ces liens peuvent se présenter sous plusieurs aspects que nous résumons ainsi :

1. l'évolution de la figuration graphique vers des formes plus claires (au cours de l'apprentissage) s'accompagne de la réussite à l'épreuve du va-et-vient (troisième expérience);

2. la clarté de la figuration graphique correspond au niveau d'explication : plus la figuration est claire, plus les explications sont formalisées ;

3. le tâtonnement précède la réussite.

Les deux premiers aspects ont une importance capitale.

93

L'étude plus fine de la figuration graphique montre que son évolution est liée à l'analyse et à la bonne compréhension des caractéristiques d'abord spatiales, ensuite techniques du matériel. La grille de cotation utilisée (cf. cotation des résultats - item figuration graphique) a permis d'établir une échelle hiérarchique. Le scalogramme obtenu (Cazalet, 1984, pp. 123-126) montre que les représentations des fonctions techniques reposent sur des outils cognitifs relatifs à l'espace et à sa représentation. Il n'est pas étonnant que les registres explicatifs qui expriment les mêmes représentations soient en correspondance avec le niveau de clarté de la figuration. L'activité des sujets, verbale et/ou figurative, autorise la dissociation des structures du réel : actions, objets. Symboliser le réel, c'est désigner, repérer, indiquer les éléments du réel sur lesquels le sujet n'a pas encore nécessairement porté son intérêt, son attention, sa conscience. Ces éléments auraient pu rester plus longtemps négligés s'il n'y avait eu cette activité de désignation, de repérage, de dissociation. On conçoit aisément que la polymorphie des moyens de désignation (graphisme et verbalisations), favorise davantage la prise en considération de ces éléments.

En somme, utiliser des moyens d'expression différents à propos d'un même objet, d'une même action, d'un même résultat, contribue à favoriser les processus de conceptualisation. Dans ces conditions, le tâtonnement et la réussite pratique sont des manifestations du retour efficace sur l'action des processus de conceptualisation.

En tâtonnant, le sujet concrétise ses connaissances abstraites, exerce un autocontrôle sur ses actions, vérifie ou non ses hypothèses (pratiques ou théoriques) et conceptualise ce qu'il fait. 

nombreux sujets se sont obligés à utiliser des symboles normalisés mal connus symbolisation que l'enseignement scientifique et technologique leur avait montré comme obligatoire. Leurs réussites sont bien moins fréquentes que chez les sujets de la situation contrôle - sans intermédiaire graphique - (résultats des deux premières expériences, voir paragraphe 3.4.).

L'utilisation "forcée » de symboles mal connus, et donc extérieurs aux sujets, semble avoir perturbé le démembrement et la reconnaissance des éléments du réel. Ce risque de perturbation introduit par l'intermédiaire graphique a été dénoncé par plusieurs auteurs, notamment Pinol-Douriez à propos de situations dans lesquelles «l'enfant ne peut projeter alors dans les expressions apprises que des schèmes préopératoires » (PinolDouriez, 1975, p.21).

Perturber les processus de conceptualisation, c'est aussi perturber les processus du tâtonnement et ceux responsables de la réussite (cf. deuxième expérience où les sujets de la situation avec figuration graphique tâtonnent moins souvent et ne réussissent pas).

\section{Conclusions}

99 Du présent travail, un principe fondamental ressort : c'est l'importance de l'activité de construction propre au sujet. Et cela, tant sur le plan pratique, sur celui des représentations, mais aussi sur les plans des moyens de représentation et de leur polymorphie.

Cette polymorphie se trouve au centre de l'activité cognitive, de la construction des connaissances. Il convient de se poser alors quelques questions :

1. Quels modes de représentation? Quelles représentations intermédiaires?

2. Pour quel niveau de connaissances? Pour l'apprentissage de quels contenus? Pour développer quelles capacités?

Répondre à ces questions impose une étude des stratégies individuelles des élèves, de ce sur quoi ils achoppent ou réussissent, je veux dire une étude de la construction du domaine des connaissances considérées.

$\mathrm{Si}$ les figurations sont des moyens commodes pour traiter, traduire, conserver, transmettre des données, le présent travail met en relief quelques conditions nécessaires à leur efficacité. L'école considère encore, à tort, les figurations comme des illustrations concrètes, transparentes, éclairant spontanément la réalité...

est peut-être pas souhaitable de raisonner seulement du point de vue strict de la discipline à enseigner et des modèles de représentation proposés... Ce qu'on appelle habituellement une séance bien «menée " n'implique pas de surcroît de favoriser la construction de nouvelles connaissances efficaces et stables chez les élèves. La deuxième population - élèves des classes de quatrième - avait un an auparavant étudié le va-et-vient électrique et sa schématisation normalisée. Or seulement $30 \%$ des bons élèves (2) et (3)) de cette population réussissent après de laborieux tâtonnements et se situent dans la situation contrôle -sans obstacle graphique.

L'élaboration des outils cognitifs nécessaires au maniement des cartes et des figures, à leur utilisation efficace, est le fruit d'une longue élaboration mentale. Cela ne fait à aucun moment l'objet d'un enseignement systématique. La mise en place des codes et langages 
«figuraux » favoriserait ainsi, du moins est-ce une hypothèse que l'on peut émettre, de véritables transferts inter - trans - disciplinaires chez nos élèves.

S'engager dans cette voie - qui s'appuie sur une véritable démarche de science expérimentale - doit permettre le dépassement des discours empiristes, des polémiques stériles, et des hypothèses - didactiques ou pédagogiques - considérées trop souvent et trop vite comme spontanément vérifiées. Dotés d'un contrôle expérimental - de terrain d'une vigilance épistémologique et méthodologique, les contenus des formations initiales ou continuées pouvant alors revêtir un caractère d'authenticité - caractère qui parfois leur fait défaut - mais caractère auquel tout enseignant de base est très attaché.

Un syllabaire où la rigueur n'exclut pas la fantaisie des propos

M. G. Vaillant, M. L. Manier : Nouvelle méthode de lecture

(Paris, E. Belin, $36^{\circ}$ éd., 1909, 56 p.) p. 46

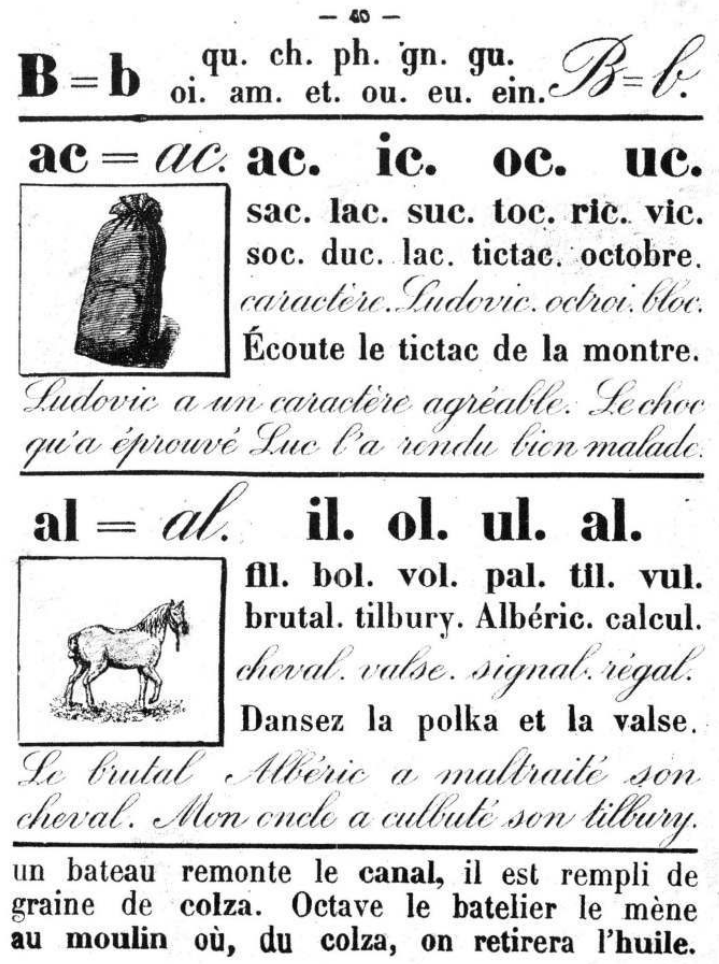

\section{BIBLIOGRAPHIE}

\section{Bibliographie}

BRESSON F « Les fonctions de représentation et de communication » in Psychologie. Encyclopédie de la Pléiade ; J. Piaget, P.Mounoud et J.P. Bronckart (Eds), Paris, Gallimard, 1987, pp.933-982. 
CAZALET E Analyse des comportements cognitifs mis en oeuvre dans la construction et l'utilisation d'un objet technique, Thèse présentée pour le Doctorat de $3^{\circ}$ cycle, Université de Provence, AixMarseille 1984.

CAZALET E., DUSSEAU J.M. « A propos de la construction d'un objet technique : le va-et-vient électrique », in Les publications de l'I.F.M., Université J. Fourier, Grenoble, 1991, pp 1-6.

HALBWACHS F. « La physique du maître entre la physique du physicien et celle de l'élève » in Revue Française de Pédagogie, $n^{\circ} 33,1975$, pp. 19-29.

INHELDER B., CELLERIER G. Le cheminement des découvertes de l'enfant, Delachaux et Niestlé, Lausanne, 1992.

JOSHUA S. «Effets comparés de la présence et de l'absence d'un schéma dans la conduite d'un raisonnement en électrocinétique ", in Bulletin de l'Union des Physiciens, $\mathrm{n}^{\circ}$ 660, 1984, pp. 523-530.

ORSINI-BOUICHOU F., HURTIG M., PAOUR J.L., PLANCHE P. « Une méthode d'apprentissage destinée à analyser les relations entre développement et fonctionnement cognitifs », in Développement et fonctionnement cognitifs chez l'enfant, G. Netchine-Grynberg (Ed), 1990, Paris, P.U.F., pp. 223-245.

PIAGET J. La prise de conscience, Paris, P.U.F., 1974a.

PIAGET J. Réussir et comprendre, Paris, P.U.F., 1974b.

PINOL-DOURIEZ M. La construction de l'espace, Neuchatel-Paris, Delachaux et Niestlé, 1975.

RABARDEL P., WEILL-FASSINA A. Le dessin technique, Paris-Londres-Lausanne, Hermès, 1987.

REY A. L'intelligence pratique chez l'enfant, Paris, Alcan, 1935.

VERGNAUD G. «Invariants quantitatifs, qualitatifs et relationnels », in Bulletin de Psychologie,tome XXVIII, n 315, 1974-75, pp. 7-8.

VERGNAUD G. : « Activité et connaissance opératoire », in Revue de l'A.P.M., 1977, pp. 52-65.

WEILL-FASSINA A. « Difficultés de l'apprentissage de la lecture du dessin technique », in Bulletin de liaison pédagogique de l'enseignement technique et de la formation professionnelle, $\mathrm{n}^{\circ} 10-11$, Paris, 1973.

\section{NOTES}

1. Le niveau scolaire choisi en référence est celui en mathématiques et en sciences physiques, avec l'avis des enseignants concernés.

2. Le niveau opératoire formel est contrôlé par l'E.C.D.L. (échelle collective de développement logique).

\section{RÉSUMÉS}

La question posée est celle de l'appropriation d'un objet technique (le va-et-vient électrique) chez l'enfant. Deux productions comportementales sont étudiées : la réalisation pratique et les 
explications données sur le fonctionnement de l'objet confectionné. On analysera donc le rôle des figurations graphiques produites par les sujets et à leur convenance. Deux situations sont aménagées : l'une avec, l'autre sans figuration graphique.

Les résultats obtenus lors des expériences préliminaires ont amené à élaborer un dispositif, d'entraînement et à évaluer ses effets. On montre comment la polymorphie des moyens d'expression peut aider les élèves.

Appropriation of a technical object (the two ways wiring) by children is studied in correspondence with two behaviours: the practical realization and the explanations about the working of the produced object. In this context, we analyse the part of graphic representations that the subject produces. Two situations are exposed: with or without graphic representation. The get results in the two preliminar experiences led us to elaborate an apprenticeship and to an evaluation of its effect. Interpretation shows how polymorphia of the means of expression can help students.

\section{INDEX}

Mots-clés : figuration graphique, réalisation, va et vient électrique

Keywords : graphic representation, realization, two ways wiring

\section{AUTEUR}

\section{ERICK CAZALET}

Enseignant au collège A. Bigot de Nîmes, chargé de cours aux universités Montpellier II, Montpellier III et à l'IUFM 